\title{
TYPICALLY REAL FUNCTIONS AND TYPICALLY REAL DERIVATIVES
}

\author{
S.Y. TRIMBLE \\ Department of Mathematics \\ University of Missouri-Rolla \\ Rolla, Missour 165401 U.S.A. \\ and \\ Texas Tech University \\ Lubbock, Texas 79409 U.S.A. \\ (Received September 19, 1985)
}

ABSTRACT. Sufficient conditions, in terms of typically real derivatives, are given which force functions to be univalent.

KEY WORDS AND PHRASES. Univalent functions and typically real functions. 1980 AMS (MDS) SUBJECT CLASSIFICATION CODE. $30 \mathrm{C} 45$.

1. TYPICALLY REAL FUNCTIONS WITH A TYPICALLY REAL FIRST DERIVATIVE.

Let $D=\{z:|z|<1\}$. Rogosinski [1] defined the class, T, of typically real functions as follows: If $f \in T$, then $f$ is regular on $D, f(z)=z+a_{2} z^{2}+\cdots$, and $\operatorname{Im}(z)$ $=0$ if and only if $\operatorname{Im}\{f(z)\}=0$. (See Goodman [2], p. 184.) The last part of this definition is equivalent to the statement that $\operatorname{Im}\{z\} * 0$ if and only if $\operatorname{Im}\{z\} \operatorname{Im}\{f(z)\}>0$. If $f \varepsilon T$, then $f$ must be one-to-one on the real interval, $(-1,1)$. So, if $f \varepsilon T$, if $z, z^{\prime} \in D$ with $z z^{\prime}$, and if $f(z)=f\left(z^{\prime}\right)$, then $\operatorname{Im}\{z\} \operatorname{Im}\left\{z^{\prime}\right\}>0$. These establish the following:

LEMMA 1. Let $f \in T$. Let $D^{+}=D \cap\{z: \operatorname{Im}\{z\}>0\}$ and $\operatorname{let} D^{-}=D \cap\{z: \operatorname{Im}\{z\}<0\}$. Then $f$ is univalent on $D$ if and only if $f$ is univalent on each of $D^{+}$and $D^{-}$ separately.

The notion of a function which is typically real on $D$ has nothing to do with its normalization. In what follows, it is convenient to say that a function, 8 , regular on $D$, is typically real on $D$ if the following holds: $\operatorname{Im}\{z\}=0$ if and only if $\operatorname{Im}\{g(z)\}=0$. This is equivalent to saying that $g$ is typically real on $D$ provided that, for $x \in(-1,1)$ and for $z \in D$, then $\operatorname{Im}\{z\} * 0$ if and only if $g^{\prime}(x) \operatorname{Im}\{z\} \operatorname{Im}\{g(z)\}>0$. 
As is known, it is not necessarily the case that a function in $T$ is univalent on $D$, e.g., $f(z)=z+z^{3}$. The following will show, however, that a simple additional requirement on functions in $\mathrm{T}$ will insure such univalence.

DEFINITION 1. Let $T^{\prime}=\left\{f \in T: f^{\prime}\right.$ is also typically real on $\left.D\right\}$.

Barnard and Suffridge [3] have shown that if $f(z)=z+a_{2} z^{2}+\cdots \varepsilon T^{\prime}$, then $\left|a_{2}\right|$ $\leq(3 \pi+2) / 2 \pi=1.8183 \cdots$ and that the result is sharp. We show the following:

THEOREM 1. If $f \varepsilon T^{\prime}$, then $f$ is univalent in $D$.

PROOF. It is enough to show that $f$ is univalent in each of $D^{+}$and $D^{-}$as defined in Lemma 1. Since $f^{\prime}$ is typically real in D it follows that $f^{\prime \prime}(0) \operatorname{Im}\left\{f^{\prime}(z)\right\}$ $>0$ for $z \in D^{+}$. Hence, $f^{\prime}$ maps the convex set, $D^{+}$, into a half-plane whose boundary passes through the origin. By a result of Noshiro [4] and of Warschawski [5], $f$ is univalent on $D^{+}$. (See Goodman [2], p. 88.) Similarly, $f$ is also univalent on $D^{-}$.

2. TYPICALLY REAL FUNCTIONS, ALL OF WHOSE DERIVATIVES ARE UNIVALENT.

In [6], Shah and Trimble introduced the class, $E$, of functions, normalized in $D$, such that $f \in E$ if and only if $\mathrm{f}^{(n)}$ is univalent in $D$ for $n=0,1,2, \ldots$ ([7] provides a survey of results about E.) Among other things, they showed that if $f \in E$, then $f$ is entire. Here, we wish to study results about functions in $E$ which are typically real.

DEFINITION 2. Let ER be those functions in $E$ such that if $f(z)=z+a_{2} z^{2}+\cdots$, then $a_{n}$ is real for $n=2,3, \cdots$. Let $\overline{E R}$ be those functions which are uniform 1 imits on compact subsets of $D$ of sequences in ER. Let ERP be those functions in ER such that $a_{n}>0$ for $n=2,3, \cdots$.

THEOREM 2. fEER if and only if $\mathrm{f}^{(n)}$ is typically real on $D$ for $n=0,1,2, \cdots$. PROOF. If every $f^{(n)}$ is typically real on $D$, then Theorem 1 implies that each $f^{(n)}$ is univalent on $D$. Hence, $f \in E R$.

On the other hand, if a function, univalent on $D$, has real Maclaurin coefficients, it is well-known that the function is typically real on $D$. Hence, if $f \varepsilon E R$, then $f^{(n)}$ is typically real on $D$ for $n=0,1,2, \cdots$.

LEMMA 2. $\overline{E R}$ - ER is the set of polynomials with real Maclaurin coefficients such that each derivative of each polynomial including the polynomial itself, is either constant or univalent on $D$.

PROOF. Let $f \in(\overline{E R}-E R)$. Then there is a sequence, $\left\{f_{k}\right\}_{k=1}^{\infty}$, in ER which converges to $f$ uniformly on compact subsets of $D$. Since the Maclaurin coefficients of each $f_{k}$ are real, the Maclaurin coefficients of $f$ must also be real. If $n \in\{0,1,2, \cdots\}$, then $\left\{f_{k}^{(n)}\right\}_{k=1}^{\infty}$ converges to $f^{(n)}$ uniformly on compact subsets of $D$. By Hurwitz's Theorem, $\mathrm{f}^{(n)}$ is either univalent or constant on $D$. If $\mathrm{f}^{(n)}$ is univalent on $D$ for all $n$, then $f \varepsilon E$, which is impossible. Hence, there is some $N$ such that $f^{(N)}$ is constant on $D$. So, if $n>N, f^{(n)}(z) \equiv 0$ on $D$. It follows that $f$ is a polynomial of degree at most $N$. 
Now let $P$ be a polynomial with real Maclaurin coefficients such that each derivative of $P$, including $P$ itself, is either constant or univalent on $D$. For $k \varepsilon\{1,2, \cdots\}$, let $r_{k}=1-1 /(k+1)$. Let $g(z)=\left(e^{\pi z}-1\right) / \pi$. (Note that $\left.g \varepsilon E R P.\right)$ Let $N$ be the degree of $F$. Let $\left\{\delta_{k}\right\}_{k=1}^{\infty}$ be a sequence of positive numbers tending monotonically to 0 . Define

$$
F_{k}(z)=\frac{P\left(r_{k} z\right)+\delta_{k} g(z)}{r_{k}+\delta_{k}} .
$$

Then $\left\{F_{k}\right\}_{k=1}^{\infty}$ converges to $F$ uniformly on compact subsets of $D$. We now show that $F_{k} \in E R$ for all $k$.

The Maclaurin coefficients of each $F_{k}$ are all real, so it is sufficient to show that, if $k \in\{1,2, \cdots\}$ and if $n \in\{0,1,2, \cdots\}$, then $F_{k}^{(n)}$ is univalent on $D$. If $n>N$, then $F_{k}^{(n)}(z)=\delta_{k} g^{(n)}(z) /\left(r_{k}+\delta_{k}\right)$, which is univalent on $D$. Since $r_{k}{ }^{N}{ }^{(N)}(z) /\left(r_{k}+\delta_{k}\right)$ is constant, $F_{k}^{(N)}$ is also univalent on D. Suppose $n<N$. To show that $F_{k}^{(n)}$ is univalent on $D$, it is enough to show that, if $0<\rho<1$, then $F_{k}^{(n)}$ is one-to-one on $\{z:|z|=\rho\}$. Let $0<\rho<1$ and let $|z|=|\omega|=\rho, z * w$. Recall that, if $h$ is univalent on $D$, then

$$
\begin{gathered}
\left|\frac{h(z)-h(\omega)}{z-\omega}\right| \geq \frac{1-\rho^{2}}{\rho^{2}} \frac{|(h(z)-h(0))(h(\omega)-h(0))|}{\left|h^{\prime}(0)\right|} \\
\geq \frac{\left|h^{\prime}(0)\right|(1-\rho)}{(1+\rho)^{3}} .
\end{gathered}
$$

(See Duren [8], p. 127.) So,

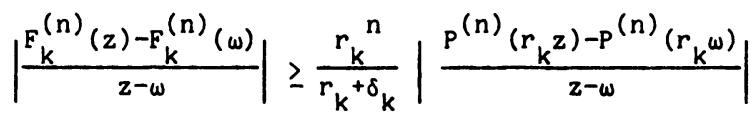

$$
\begin{aligned}
& -\frac{\delta_{k}}{r_{k}+\delta_{k}}\left|\frac{g^{(n)}(z)-g^{(n)}(w)}{z-\omega}\right| \\
& >\frac{(1 / 2)^{N}}{1+\delta_{1}} \frac{\left|p^{(n+1)}(0)\right|(1-0)}{(1+p)^{3}}-\frac{\delta_{1}}{(1 / 2)} \max _{|\zeta|=\rho}\left|g^{(n+1)}(\zeta)\right| \\
& \geq \frac{(1 / 2)^{N}}{1+\delta_{1}} \frac{\left|P^{(n+1)}(0)\right|(1-0)}{(1+p)^{3}}-2 \delta_{1} \pi^{N} e^{\pi}
\end{aligned}
$$

Choose $\delta_{1}$ so that this last expression is positive for $0 \leq n<N$. Then $F_{k}^{(n)}$ will be one-to-one on $\{z:|z|=\rho\}$. The proof of the lemma is done. 
In what follows, it is convenient to write functions in $\overline{E R}$ as $z+\sum_{k=2}^{\infty} b_{k} z^{k}$, even though some of them may be polynomials.

THEOREM 3. Let $f \in E R$ and $g \in \overline{E R}$. Let $\lambda \varepsilon(0,1)$. Suppose $f(z)=z+\sum_{k=2}^{\infty} a_{k} z^{k}$ and $g(z)=z+\sum_{k=2}^{\infty} b_{k} z^{k}$. Assume $a_{k} b_{k} \geq 0$ for all $k$. If $h(z)=\lambda f(z)+(1-\lambda) g(z)$, then $h \in E R$. Hence, ERP is a convex set.

PROOF. Since $a_{k} b_{k} \geq 0$, the signs of $n^{(n+1)}(0), f^{(n+1)}(0)$, and $g^{(n+1)}(0)$ are all the same. So, if $z \in D, h^{(n+1)}(0) \operatorname{Im}\{z\} \operatorname{Im}\left\{h^{(n)}(z)\right\}=\lambda h^{(n+1)}(0) \operatorname{Im}\{z\} \operatorname{Im}\left\{f^{(n)}(z)\right\}+$ $(1-\lambda) h^{(n+1)}(0) \operatorname{Im}\{z\} \operatorname{Im}\left\{g^{(n)}(z)\right\}>0$ if and only if $\operatorname{Im}\{z\} * 0$. Hence, $h^{(n)}$ is typically real on $D$. By Theorem 2, heER. If $f, g \in E R P$, then $a_{k} b_{k}>0$ and so $[\lambda f+$ $\left.(1-\lambda)_{g}\right] \varepsilon$ ERP, 1.e., ERP is convex.

REMARK. Suffridge [9] has shown that, if $f \in E R P$ and if $f(z)=z+a_{2} z^{2}+\cdots$, then $a_{2 k+1} \leq \pi^{2 k} /(2 k+1) !$ for $k=1,2, \cdots$ and $a_{2 k} \leq 2 a_{2} \pi^{2(k-1)} /(2 k) !$. The inequalities are sharp. It is interesting that $a_{2}$ is necessarily involved in the bounds for the even coefficients but not for the odd.

\section{REFERENCES}

1. Rogosinski, W. Uber positive harmonische Entwicklungen und typischreele Potenzreihen, Math. Ze1t. 35 (1932) 93-121.

2. Goodman, A.W. Univalent Functions, Vol. I, Mariner Publishing Company, Tampa, Florida, 1983.

3. Barnard, R.W. and T.J. Suffridge On the Simultaneous Univalence of $f$ and $f^{\prime}$, Michigan Math. J. 30 (1983) 9-16.

4. Noshiro, K. On the Theory of Schlicht Functions, J. Fac. Sci. Hokkaido Univ. Jap (1) 2 (1934-1935) 129-155.

5. Warschawski, S. On the Higher Derivatives at the Boundary in Conformal Mapping, Trans. Amer. Math. Soc. 38 (1935) 310-340.

6. Shah, S.M. and S.Y. Trimble Univalent Functions with Univalent Derivatives, Bull. Amer. Math. Soc. 75 (1969) 153-157; Erratum, ibld 75 (1969), 888.

7. Shah, S.M. and S.Y. Trimble Analytic Functions with Univalent Derivative, Indian J. Math. 20 (1978) 265-299.

8. Duren, P.L. Univalent Functions, Springer-Verlag, New York, 1983.

9. Suffridge, T.J. Analytic Functions with Univalent Derivatives, Ann. Univ. Mariae Curie-Sklodowska Sect. A 36/37 (1982/1983) 143-148. 


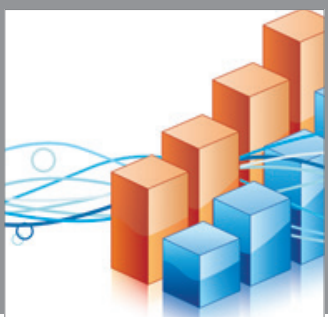

Advances in

Operations Research

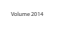

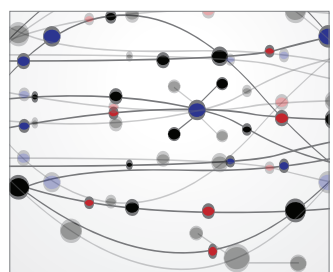

\section{The Scientific} World Journal
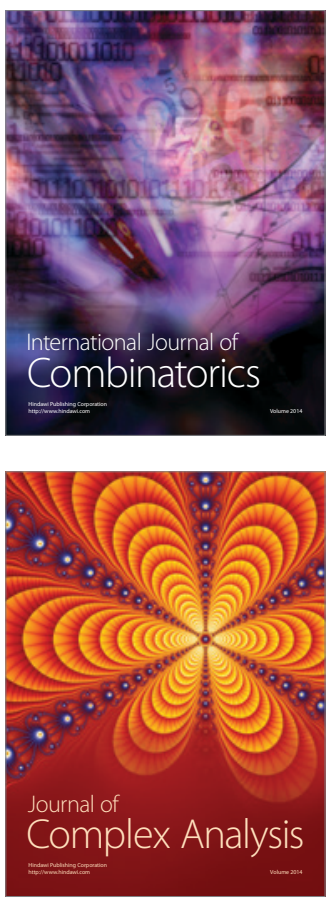

International Journal of

Mathematics and

Mathematical

Sciences
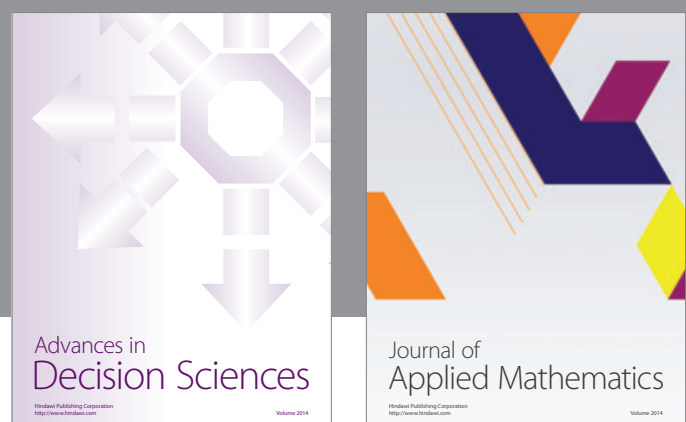

Journal of

Applied Mathematics
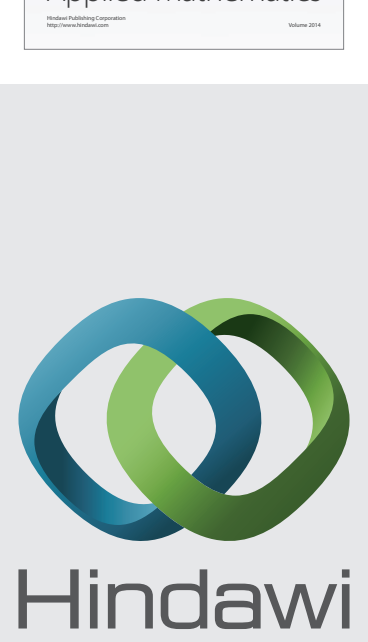

Submit your manuscripts at http://www.hindawi.com
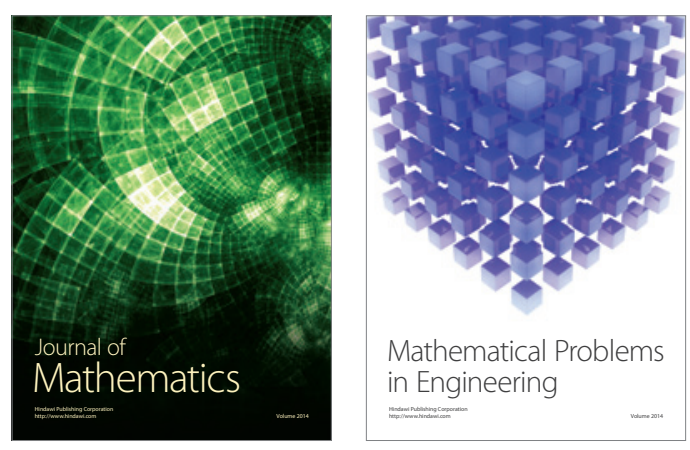

Mathematical Problems in Engineering
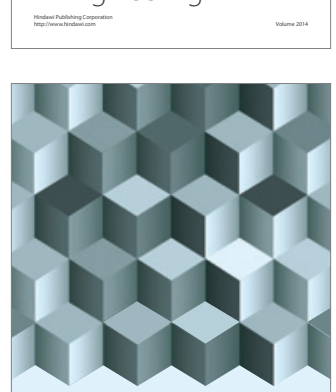

Journal of

Function Spaces
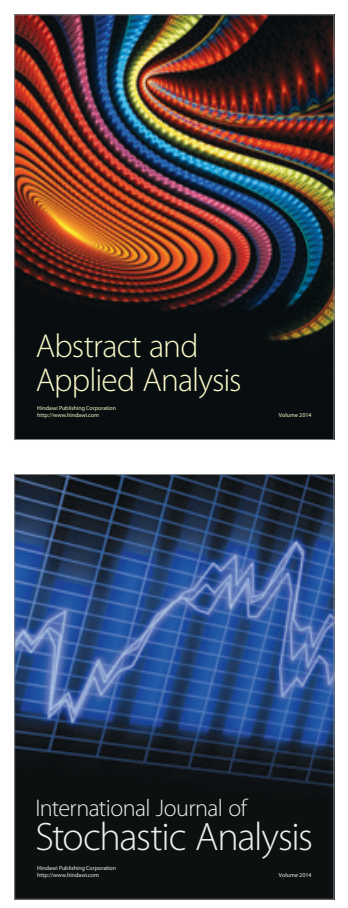

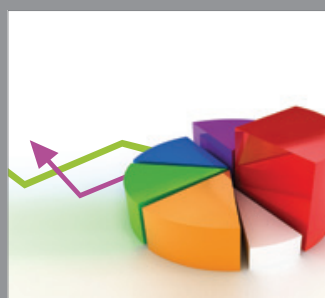

ournal of

Probability and Statistics

Promensencen
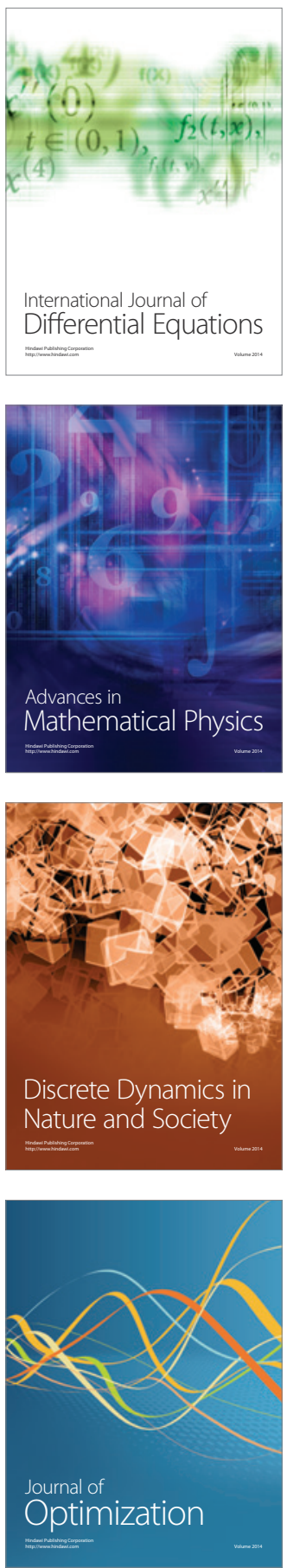